\title{
Congenital dermoid cysts of the anterior fontanel
}

Sir,

The congenital dermoid inclusion cyst (CDIC), the most common type of dermoid cyst of the head and neck, ${ }^{[1]}$ is a soft mobile cystic mass covered by normal skin which does not cause any discomfort, pain, or throbbing. There is no communication between the cyst and the intracranial cavity. ${ }^{[2]} \mathrm{A}$ dermoid cyst is the most common lesion found around the anterior fontanel. ${ }^{[3]}$ However, CDIC of the anterior fontanel is a rare lesion accounting for $0.2 \%$ of all inclusion cysts ${ }^{[4]}$ and $0.1-0.5 \%$ of all cranial tumors. ${ }^{[1]}$

Here we describe eight cases of CDIC of the anterior fontanel, emphasizing the importance of early diagnosis and treatment of these lesions.

Eight patients referred to the Children's Hospital Medical Centre in Tehran from March 2000 to 2005 for the surgical resection of dermoid cysts around the anterior fontanel.
In all cases [Table 1], the lesion was seen at birth as a soft tissue mass around the anterior fontanel, and presented as a firm mobile tumour with progressive growth but no neurological findings. In six cases, the lesion was on the left side. Seven lesions were at the lateral angle, only one was at the anterior angle of the anterior fontanel. The maximum diametres of the lesions varied from $24-54 \mathrm{~mm}$ respectively (mean $=36 \mathrm{~mm}$ ). One patient had an infected dermoid cyst with inflamed, red, and tender skin over the lesion whereas the others had an intact skin covering the lesion [Figure 1]. All patients underwent preoperative neuroimaging: CT scans showed an extracranial heterogeneous hypodense mass and MRI a hypointense lesion on T1-weighted [Figure 2] as well as a hyperintense lesion on T2-weighted images. In four patients, there was a distinct lytic bone lesion under the cyst without any intracranial extension.

Patients were operated in the supine position, under sedation and local anaesthesia. The lesion was completely removed through an ellipsoid incision bordering the mass, which was easily dissected from the galea and periosteum, without damage to the sagital sinus or the cyst capsule. Histological investigation showed cysts lined by a thick, stratified squamous epithelium containing skin appendages (hair follicles, sebaceous glands, and sweat glands) in all cases. The postoperative period was uneventful with no recurrence during the follow-up period of $1.5-6$ years (mean $=3.2$ years) [Figure 3 ].

Dermoid cyst is a pathologic term for a cyst lined by squamous epithelium containing skin appendages (hair follicles, sebaceous, and sweat glands). It has been classified into three categories: (1) Teratoma type congenital dermoid cysts, (2) Acquired implantation dermoid cysts, (3) CDIC resulting from the inclusion of displaced dermoid cells along the embryonic fusion line. ${ }^{[2]}$ Dermoid cysts of the head and neck are thought to be mostly of the CDIC type, which is usually around the anterior fontanel. ${ }^{[1]}$

CDIC of the anterior fontanel is described as a slowgrowing, nontender, soft lump covered with intact skin. These are usually observed at birth and develop gradually through he accumulation of secretions and internal desquamation. ${ }^{[4]}$

In spite of the presentation of this cyst at birth, the age of children admitted for surgery in this study ranges from two months to five years. Postponing the operation in 
Table 1: Summary of the clinical, demographic, and neuroimaging characteristics of the studied cases

\begin{tabular}{|c|c|c|c|c|c|c|c|}
\hline Patient no & Sex & Age & Side & Site & Neuro-imaging & Bone defect & Skin \\
\hline 1 & Female & 2 months & Left & Lateral & MRI & - & Infected \\
\hline 2 & Male & 4 years & midline & Anterior & MRI & + & Intact \\
\hline 3 & Male & 4 months & Left & Lateral & $\mathrm{CT}$ & - & Intact \\
\hline 4 & Male & 5 years & Left & Lateral & MRI & + & Intact \\
\hline 5 & Female & 1 year & Left & Lateral & MRI & + & Intact \\
\hline 6 & Female & 4 months & Left & Lateral & CT & - & Intact \\
\hline 7 & Female & 3 months & Left & Lateral & MRI & - & Intact \\
\hline 8 & Male & 1 year & Right & Lateral & $\mathrm{CT}$ & + & Intact \\
\hline
\end{tabular}

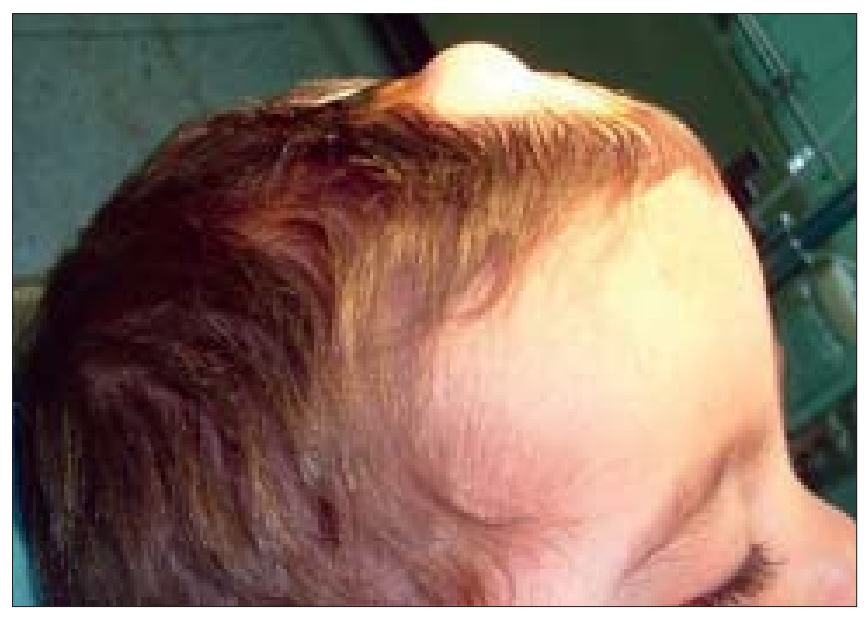

Figure 1: The child with lump around the anterior fontanel

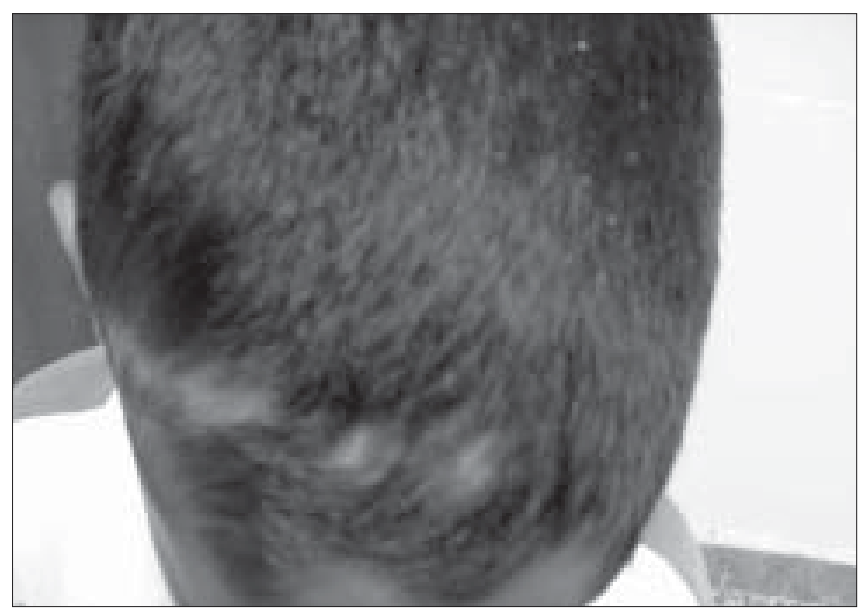

Figure 3: Photography of patient two years after surgery

some cases can be related to the parents' ignorance or their knowledge of the benign nature of the lesion that permits delayed surgery. Although a female predominance has been reported, ${ }^{[4]}$ we could not find any gender difference. The size of the dermoid cyst has usually been $1-7 \mathrm{~cm}$ in the previously reported cases,,$^{[4]}$ corresponding to the age of the patient at the time of the diagnosis. ${ }^{[2]}$

These cysts arise mostly around the anterior angle of the fontanel. ${ }^{[2,5]}$ Interestingly, most lesions (6/8) in this series were at the left lateral angle of the anterior fontanel.

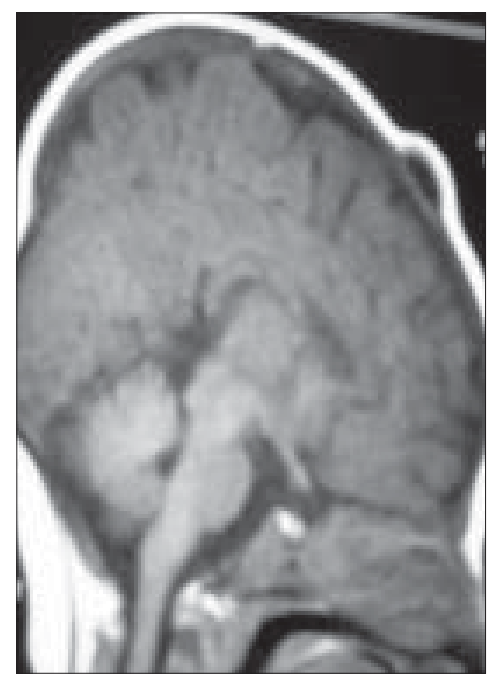

Figure 2: T1-Weighted image, sagittal view of brain MRI of a two month-old infant reveals hypointense mass around the anterior fontanel with intact bone under the lesion

This kind of different localization is probably due to the geographic differences.

Most lesions found around the anterior fontanel in infancy are dermoid cysts. ${ }^{[3]}$ Other important pathologies are encephalocoele, meningocoele, sebaceous cyst, lipoma, haemangioma, and cephalohematoma. ${ }^{[1,2,4,6]}$ Precise physical examination and neuroimaging can be useful for differential diagnosis

Computed tomography (CT) scans demonstrate the lesion as an extracranial, encapsulated, low-density mass without enhancement (known as a fluid-containing cyst) that is sometimes accompanied by lytic bone lesions. ${ }^{[2,7]}$ Magnetic Resonance Imaging (MRI) is the most reliable and accurate test ${ }^{[4]}$ and shows the lesion as a low- and highintensity mass on T1-weighted and T2-weighted images, respectively. ${ }^{[3,7-9]}$ It has been recommended to perform CT or MRI before surgery ${ }^{[9]}$ to determine the extension of the cyst and the presence of a lytic bone lesion.

Although Chaudhari et al. found no direct relationship 
between the depth of the bone defect and time of presentation, ${ }^{[7]}$ all the patients diagnosed during the first year of life (four patients) showed no lytic bone lesion in their imaging in this study. These findings emphasize the importance of an early diagnosis and intervention. Thus, we do not recommend any neuroimaging for mobile cystic masses placed around the anterior frontal in patients younger than a year of age.

Dermoid cysts of the anterior fontanel are excised for cosmetic reasons, to prevent infection, to obtain histological diagnoses, and to rule out malignancy. ${ }^{[3,7-9]}$ The best procedure is a complete resection of the cystic mass with removal of the wall by blunt dissection of the tumour from the underlying tissue (dura or cranium) through an ellipsoid incision bordering the mass. ${ }^{[1]}$ Care should be taken while excising the tumour overlaying an open anterior fontanel or a lytic bone lesion ${ }^{[7]}$ to avoid injury of the underlying structures. Local rather than general anesthesia is preferred in most cases, especially when no lytic bone lesion exists, or the size is not very large.

As seen in our study, there is almost no recurrence if en bloc resection of the cyst is performed. Until now, there has been just one case of progression and slow recurrence six years after surgery. ${ }^{[4]}$

\section{Masood Majed, Farideh Nejat, Mostafa El Khashab}

Children's Hospital Medical Center, Tehran University of Medical Sciences, Tehran, Iran.

\author{
Address for Correspondence: Farideh Nejat, \\ Children's Hospital Medical Center, \\ Tehran University of Medical Sciences, Tehran, Iran. \\ E-mail: nejat@sina.tums.ac.ir
}

\section{REFERENCES}

1. Aslan Ö. Congenital dermoid cyst of the anterior fontanelle in Turkish children-four case reports. Neurol Med Chir (Tokyo) 2004;44:150-2.

2. Aquino $\mathrm{H}$. Congenital dermoid inclusion cyst over the anterior fontanel. Arq Neuropsiquiatr 2003;61:448-52.

3. Martinez-Lage J. Cranial fasciitis of the anterior fontanel. Child's Nerv Syst 1997;13:626-8.

4. Carvalho GT, Fagundes-Pereyra WJ, Marques JA, Dantas FL, Sousa AA. Congenital inclusion cysts of the anterior fontanelle. Surg Neurol 2001;56:400-5

5. Gumerlock MK. Epidermoid, dermoid, and neurenteric cysts in youmans neurological surgery. $5^{\text {th }}$ ed. Philadelphia: W.B. Suanders; 2004. p. 1223-30.
6. Fermin S. Congenital dermoid cyst of the anterior fontanel in mestizo-mulatto children. Childs Nerv Syst 2001;17:353-5.

7. Hashiguchi K. Subgaleal dermoid tumors at the anterior fontanelle. Pediatr Neurosurg 2005;41:54-7.

8. Shepherd G. Pediatric dermoid cysts of the head and neck. Otolaryngol Head Neck Surg 2005;132:938-42.

9. Tateshima S. Rapidly enlarging dermoid cyst over the anterior fontanel: A case report and review of the literature. Childs Nerv Syst 2000;16:875-8. 